\title{
Outcome of Self- and Planned Extubation in Organophosphate-Poi- soned Patients
}

\author{
Abbas Aghabiklooei ${ }^{1}$, Omid Mehrpour ${ }^{2}$, Hossein Hassanian-Moghaddam ${ }^{3,4}$, Shahin Shadnia ${ }^{3,4}$ and \\ Nasim Zamani ${ }^{3,4 *}$
}

${ }^{1}$ Department of Legal Medicine and Toxicology, Firoozgar Hospital, Iran University of Medical Sciences, Iran

${ }^{2}$ Faculty of Medicine, Birjand University of Medical Sciences, Iran

${ }^{3}$ Department of Clinical Toxicology, Loghman-Hakim Hospital, Iran

${ }^{4}$ Excellence Center of Clinical Toxicology, Iranian Ministry of Health, Iran

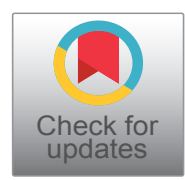

*Corresponding author: Nasim Zamani, MD, Department of Clinical Toxicology, Loghman Hakim Hospital, South Karegar Street, Tehran, Iran, Tel/Fax: 00982155424041

\begin{abstract}
Background: Respiratory failure is the most common cause of morbidity and mortality in organophosphate (OP)-intoxicated patients. We aimed to assess and compare the need for re-intubation and outcome between patients with self-extubation (SE) and planned extubation (PE).

Methods: All OP-poisoned endotracheally intubated patients admitted to poisoning ICU were included. The frequency and time of SE, need for re-intubation, and its impact on hospital stay and outcome were assessed.

Results: In fifteen patients (48.4\%) SE was reported. Need for re-intubation in these patients was more than those who underwent $P E(60.0 \%$ vs. $37.5 \% ; P=0.2)$. Early unplanned SE significantly correlated with occurrence of pulmonary complications $(P=0.04)$. The rate of aspiration pneumonia was high $(80 \%)$ in SE cases. Hospital stay was also significantly prolonged in these patients (14.6 vs. 5.4 days, $\mathrm{P}=0.04$ ).

Conclusion: Planning for on-time weaning/extubation in OP-poisoned patients can prevent unplanned SE and decrease the occurrence of lung complications.
\end{abstract}

\section{Keywords}

Insecticide, Organophosphate, Poisoning, Extubation, Outcome

\section{Objectives}

World Health Organization defines pesticides as chemical compounds used to kill pests, including insects, rodents, fungi, and unwanted plants. Pesticides are grouped based on their composition into carbamates, organochlorines, and organophosphates [1]. The use of pesticides benefits agricultural productivity and public health. Pesticides play a significant role in controlling vector-borne diseases, which are a main public health concern [2]. Organophosphates and carbamates are widely used as insecticides which inhibit cholinesterase activity [3].

Respiratory failure frequently occurs after severe organophosphate (OP) insecticide poisoning [4]. Most of OP-poisoned patients need tracheal intubation and mechanical ventilation (MV) for respiratory support. Pulmonary complications including bronchospasm, bronchorrhea, respiratory muscle weakness, pulmonary edema, pneumonia, and hypoxia are the most common causes of morbidity and mortality in these patients $[5,6]$. Weakness of the respiratory muscles may last for a long time if acetylcholinestrase (AchE) is irreversibly blocked by OPs.

On time weaning and extubation is very important in these patients while unplanned self-extubation (SE) is a serious health care concern and is an indicator of poor quality and safety of care [7]. Early SE at inappropriate time may cause respiratory failure and need for re-intubation with its possible complications such as aspiration pneumonia [8].

The re-intubation risk in general intensive care units

Citation: Aghabiklooei A, Mehrpour O, Hassanian-Moghaddam H, Shadnia S, Zamani N (2018) Outcome of Self- and Planned Extubation in Organophosphate-Poisoned Patients. Int J Anesthetic Anesthesiol 5:075. doi.org/10.23937/2377-4630/1410075

Accepted: October 22, 2018: Published: October 24, 2018

Copyright: ( 2018 Aghabiklooei A, et al. This is an open-access article distributed under the terms of the Creative Commons Attribution License, which permits unrestricted use, distribution, and reproduction in any medium, provided the original author and source are credited. 
(ICUs) varies between 2 to 25 percent [5]. Almost 20\% of the re-intubations happen within the first 72 hours after extubation [6]. Inadequate sedation as well as agitation is a major risk factor for SE. Need for re-intubation is the major determinant of the patient's outcome. Both SE and re-intubation can be followed by serious complications mainly aspiration, laryngeal edema, and increased risk for pneumonia [9]. Most of the related studies have been performed in the surgical and general ICUs. We aimed to evaluate the frequency of SE and its failure rate, causes of need for re-intubation, and outcome in the OP-poisoned patients. We also aimed to compare the outcome between the patients who were planned to be extubated and those who self-extubated.

\section{Methods}

\section{Patients and diagnostic inclusion criteria}

In a prospective cross-sectional survey, all severely OP-poisoned patients older than 14 years who were brought to poisoning emergency department (ED) and had undergone tracheal intubation and been admitted to the adult poisoning ICU of our center between March 2013 and March 2014 were included. In our center, poisoned children and adolescents younger than 14 years are admitted to PICU.

Patients younger than 14 years and those with mixed toxicity, toxicity with insecticides other than OPs, accidental unplanned extubation due to tracheal tube displacement, re-intubation due to tube obstruction, and patients with underlying cardiovascular or lung diseases were excluded.

Diagnosis was made by positive history of exposure to OPs and development of cholinergic syndrome and was confirmed by decreased butyrylcholinesterase (below lower normal limits or decreased more than $25 \%$ compared to the first level available). Serum level of acetylcholinesterase (AchE) was checked on presentation and daily, afterwards, during hospitalization. We differentiated OPs from carbamates by direct observation of the poison package or coverage brought by the patients' family based on the physician request. Unknown cases whose poison sample was unavailable were excluded.

Atropine and pralidoxime (2-PAM) were initiated for all patients at ED. All patients underwent gastric aspiration and washing with normal saline, received a single dose of charcoal ( $1 \mathrm{~g} / \mathrm{kg}$ ) via nasogastric tube, and were admitted to toxicology ICU. In ICU setting, all patients had physical restraint and received intravenous midazolam with the dose 2 to $5 \mathrm{mg}$ and fentanyl with the dose 25 to $50 \mu \mathrm{g}$ as needed every 4 to 6 hours to control agitation.

\section{Definitions}

Planned extubation (PE) was defined as extubation process performed after successful weaning from ven- tilator by the physician and self-extubation (SE) was defined as the endotracheal tube being removed in an unplanned manner by the patient. We used "DAS extubation guidelines" for criteria of extubation [10]. Re-intubation was defined as intubation for two or more times during the same hospitalization period.

Pulmonary complications were classified as acute respiratory distress syndrome (ARDS) and pneumonia. Aspiration pneumonia was diagnosed by observation of purulent secretion, fever (oral $\mathrm{T}>38.3^{\circ} \mathrm{C}$ ), lekucytosis, and focal air space fillings in chest radiography. ARDS was defined as hypoxia without response to oxygen therapy, diffuse crackles, bilateral diffuse patchy infiltrations in the chest radiography, and $\mathrm{FiO}_{2} / \mathrm{PaO}_{2}$ ratio $<200$. We could not assess the pulmonary capillary wedge pressure in our setting. Intermediate syndrome (IS) was defined as a state of muscle paralysis associated with relapse of cholinergic symptoms after recovery of cholinergic crisis [11].

\section{Data collection}

A self-made questionnaire containing information on the amount of the ingested OP, presenting signs and symptoms, on-arrival vital signs, on-arrival and daily lab tests, treatments given, and the patients' final outcome was filled for every single patient by trained fellows. On-arrival and before and after weaning and extubation respiratory indexes (respiratory rate/venous blood gas (VBG) analyses $/ \mathrm{O}_{2}$ saturation), signs and symptoms of respiratory distress as well as the level of consciousness based on Glasgow coma scale (GCS) were also recorded. Type of extubation (self- versus planned extubation), need for re-intubation, causes and number of re-intubation episodes, hospital stay, duration of $\mathrm{MV}$, complications, and finally, the outcome were investigated and compared between those with planned and self-extubation. If the patients needed re-intubation, the causes and clinical condition of the patients at re-intubation time were documented, as well.

\section{Statistical analysis}

Data was analyzed using statistical package for social sciences (SPSS) software version 18 and by application of Chi-Square, Fisher's exact, and Mann-Whitney $U$ tests for comparison of nonparametric variables and student $t$ test for parametric variables. $P$ values of 0.05 or less were considered to be statistically significant. This study was approved by the Ethics Committee of Shahid Beheshti University of Medical Sciences. Since the patients were intubated, informed consent was taken from their next if keen.

\section{Results}

Of 36 OP-poisoned patients who underwent tracheal intubation and were admitted to ICU, three and two were excluded because of endotracheal tube obstruction and tube displacement, respectively. Finally, 31 cases met our inclusion criteria and were enrolled. 
Table 1: Outcome of patients based on type of extubation.

\begin{tabular}{|l|l|l|l|l|}
\hline & Total N: $\mathbf{3 1}$ & Self-extubation N: 15 & Planned extubation N: 16 & P \\
\hline Re-intubation & $15(48.4 \%)$ & $9(60 \%)$ & $6(37.5 \%)$ & $0.210^{*}$ \\
\hline Lung Complications & $22(70.9 \%)$ & $12(80.0 \%)$ & $10(62.5 \%)$ & $0.25^{* *}$ \\
\hline Hospital Stay $(\mathrm{hr})$ & & $349.8 \pm 199.2(80-720)$ & $226.9 \pm 136.5(63-480)$ & $0.04^{\ddagger}$ \\
\hline Duration of MV- $(\mathrm{hr})$ & & $223.5 \pm 143.3(37-576)$ & $129.1 \pm 78.8(73-296)$ & $0.52^{\ddagger}$ \\
\hline Death Rate & $5(16.1 \%)$ & $2(13.3 \%)$ & $3(18.7 \%)$ & $0.57^{* *}$ \\
\hline
\end{tabular}

-MV = Mechanical Ventilation, " ${ }^{*}$ Fisher Exact test, ${ }^{\ddagger}$ Mann-Whitney test, ${ }^{*}$ Chi-Square.

Table 2: Correlation between re-intubation and mean Atropine, 2-PAM, and serum AchE during hospitalization.

\begin{tabular}{|c|c|c|c|c|}
\hline & & \multicolumn{2}{|l|}{ Re-intubation } & \multirow[t]{2}{*}{$\mathbf{P}$} \\
\hline & & Yes (15) & No (16) & \\
\hline \multirow[t]{2}{*}{ Serum AchE } & Mean \pm SD & $1615 \pm 2476$ & $1158 \pm 1420$ & $0.640^{\ddagger}$ \\
\hline & (range) & $(251-5323)$ & $(195-2790)$ & \\
\hline Atropine (mg/day) & Mean \pm SD & $4.6 \pm 11.3$ & $5.7 \pm 6.3$ & $0.441^{\ddagger}$ \\
\hline \multirow[t]{2}{*}{ Duration of Atropine (day) } & Mean \pm SD & $9.7 \pm 5.8$ & $5.7 \pm 5$ & $0.070^{\ddagger}$ \\
\hline & Median (range) & 10 (1 to 18$)$ & $3(1$ to 15$)$ & \\
\hline \multicolumn{5}{|l|}{ Complications } \\
\hline Pneumonia & & $13(86.6 \%)$ & $8(50 \%)$ & $0.050^{* *}$ \\
\hline ARDS & & $0(0.0 \%)$ & $1(6.2 \%)$ & \\
\hline $\mathbf{I S}^{*}$ & & $3(20 \%)$ & $2(12.5 \%)$ & \\
\hline \multirow[t]{2}{*}{ MV duration (hr) } & Mean \pm SD & $223.5 \pm 143.3$ & $103.93 \pm 74.79$ & $0.524^{\ddagger}$ \\
\hline & (range) & $(50-576)$ & $(11-240)$ & \\
\hline \multirow[t]{2}{*}{ Hospital stay (hr) } & Mean \pm SD & $349.8 \pm 199.2$ & $195.7 \pm 100.0$ & $0.135^{\ddagger}$ \\
\hline & (range) & $(70-720)$ & $(63-480)$ & \\
\hline Death rate & & $5(33.3 \%)$ & $0(0.0 \%)$ & 0.011 \\
\hline
\end{tabular}

‡Mann-Whitney test, " ${ }^{*}$ Fisher Exact test, ${ }^{*}$ IMS = Intermediate syndrome.

Mean age was $33.8 \pm 19.1$ (range; 13-77) years with a male to female ratio of 2 to 1 . Suicide attempt by oral ingestion was the cause of poisoning in all cases. Mean amount of the ingested poison was $124 \mathrm{~mL}$ (range; 10500). Mean time elapsed between ingestion of the poison and ED presentation was 2.7 hours (range; 1 to 8 ). Ten cases $(33 \%)$ underwent airway intubation in the first 4 hours of presentation and the remainder was intubated 4 to 8 hours post ED presentation. Pneumonia, IS, and ARDS occurred in 22 (70.9\%), five (16.1\%), and one $(3.2 \%)$ patients, respectively.

Self-extubation occurred in 15 cases (48.4\%), twelve of whom underwent re-intubation within the first 24 hours post extubation. Mean GCS at the time of SE was 11.8. Nine of these patients needed re-intubation because of respiratory failure. Causes of re-intubation were similar between those who self-extubated and those who were planned to be extubated. Patients who were re-intubated were not significantly different from those who were not in terms of age, gender, ingested dose, and serum AchE. Table 1 shows the patients' outcome based on type of extubation.

Fifteen cases $(48.4 \%)$ underwent re-intubation. After extubation, $17(54.8 \%)$ and $25(80.6 \%)$ still needed to receive atropine and 2-PAM. Re-intubation was statistically associated with pulmonary complications $(P=0.05)$ and only two patients with re-intubation remained without complications. On the other hand, in 8 patients who experienced complications, re-intubation happened once, twice and three times, respectively $(P$
$=0.029)$. Three $(9.6 \%)$ cases underwent tracheostomy because of prolonged MV.

Mean VBG values of the patients who underwent re-intubation were as follow: $\mathrm{PH}: 7.39 \pm 0.8$ (range; 7.237.52), $\mathrm{PCO}_{2}: 41.3 \pm 8.8$ (range; $\left.25-57\right) \mathrm{mmHg}, \mathrm{PO}_{2}: 52.2 \pm$ 14.1 (range; 33.8-83.7) mmHg, $\mathrm{HCO}_{3}: 26.1 \pm 4.7$ (range; 16.4-31.4) $\mathrm{mmol} / \mathrm{L}$, and $\mathrm{O}_{2}$ sat: $79.7 \pm 11.6$ (range; 54.596\%). Hypercapnia ( $\mathrm{PCO}_{2}>45 \mathrm{mmHg}$ ) [12] and respiratory acidosis, severe tachypnea and hyperventilation associated with respiratory distress, and severe resistant hypoxia were the main causes of re-intubation in 5, 2, and 12 patients. Nineteen of 31 patients received sedation ( 5 were on fentanyl, 8 were on midazolam, and 6 were on both). Mean doses of fentanyl and midazolam were $70 \pm 29 \mu \mathrm{g} / \mathrm{q} 4 \mathrm{~h}$ and $4.1 \pm 1.2 \mathrm{mg} / \mathrm{q} 4 \mathrm{~h}$, respectively, but almost $50 \%$ of our cases extubated themselves.

Mean hospital stay was $11.2 \pm 7.1$ (range; 2.6 to 30 ) days and mean duration of MV was 6.7 days. Total death rate was $16.1 \%$ (5 patients) with no significant difference between those with self-extubation and those with planned extubation $(P=0.57)$; however, such a significant difference was observed between those who were re-intubated and those who did not $(P=0.011$; Table 2). Mean dose of atropine used at ED was $5.18 \pm 8.96 \mathrm{mg}$. The administered 2-PAM was not significantly correlated with neither intubation period $(P=0.23, r=0.22)$ nor hospitalization period $(P=0.16, r=0.25)$. Figure 1 and Table 3 show serum AchE changes in all cases within the period of hospitalization. Mean serum level of AchE was $1915 \pm 277 \mathrm{U} / \mathrm{L}$ (range; 195 to 5358) on arrival. 


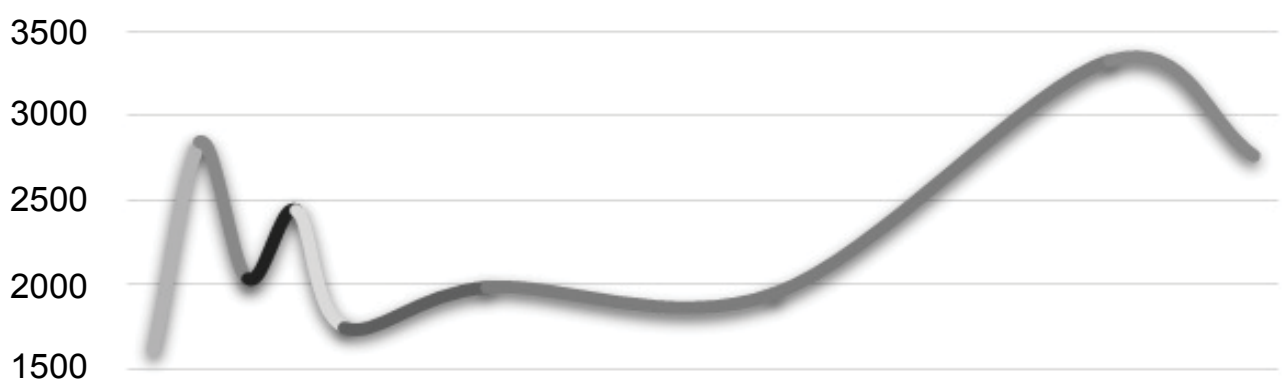

1000

500



Figure 1: Serum AchE changes during hospitalization.

Table 3: Relation between serum level of AchE and reintubation.

\begin{tabular}{|c|c|c|c|c|c|}
\hline & \multicolumn{4}{|c|}{ Re-intubation } & \multirow[t]{3}{*}{$\mathrm{P}^{\ddagger}$} \\
\hline & Yes & & No & & \\
\hline & Mean \pm SD & Median (range) & Mean \pm SD & Median (range) & \\
\hline AchE At ED & $2070 \pm 2821$ & 600 (288 to 5323$)$ & $1159 \pm 1421$ & 491 (195 to 2790 ) & 0.513 \\
\hline AchE $2^{\text {nd }}$ day & $1414 \pm 2920$ & 427 (200 to 8637$)$ & $4466 \pm 3543$ & 6167 (62 to 8694$)$ & 0.298 \\
\hline AchE $3^{\text {rd day }}$ & $901 \pm 1051$ & 460 (200 to 2760$)$ & $3162 \pm 3391$ & 1080 (641 to 8231$)$ & 0.047 \\
\hline AchE $4^{\text {th }}$ day & $382 \pm 207$ & 393 (170 to 571$)$ & $3575 \pm 2784$ & 4911 (375 to 5440$)$ & 0.157 \\
\hline AchE $5^{\text {th }}$ day & $1148 \pm 1218$ & 722 (142 to 3695$)$ & $3035 \pm 2166$ & 3435 (242 to 5596$)$ & 0.062 \\
\hline
\end{tabular}

AchE = Acetylecholinestrase, $\ddagger^{\ddagger}$ Mann-Whitney test.

\section{Discussion}

Although exposure to OPs has significantly decreased after 1995 in the US, it is still one of the most important causes of insecticide toxicity in most countries [13]. Patients with severe OP poisoning may develop respiratory failure and most of them will need tracheal intubation and MV [11]. Unplanned SE and re-intubation can be followed by serious complications including aspiration, laryngeal edema, and increased risk for pneumonia [9]. Many SEs result in failure within the first 72 hours [14-16] while re-intubation is a major determinant of the patient outcome.

Unfortunately, no standard test is available to predict the appropriate time for extubation $[14,15]$. SE occurred in nearly $50 \%$ of our patients, $60 \%$ of whom underwent re-intubation. This means a high failure rate of SE in our patients which may be due to ongoing respiratory failure because of respiratory muscle weakness secondary to OP effects. Self-extubation rate was very high (nearly $50 \%$ ) in comparison to other studies. SE rate was 4 to $15 \%$ in internal medicine ICUs [16]. This difference may be due to severe agitation in the OP-poisoned patients probably due to the effects of atropine.

Some researchers have shown that unplanned SE may accompany with complications and a poor prognosis [17-19]. In our study, pneumonia was the most common complication and its occurrence was not significantly different between the SE and PE groups.

\section{Risk factors of extubation failure and re-intubation}

Re-intubation is accompanied by a 5 -fold mortality and 2-fold longer hospitalization period [6]. In our study, nearly $60 \%$ of those with SE underwent re-intubation, an almost 2-fold rate compared to those with planned extubation (37\%). Multiple re-intubations may lead to difficult re-intubation with a higher mortality rate, as well [6]. Factors including visiting the patient by different physicians, young age, age over 70 , long-term MV, long-term use of sedatives, and hemoglobin less than $10 \mathrm{~g} / \mathrm{dL}$ or hematocrit less than $30 \%$ at extubation time may increase the risk of re-intubation [5]. In this study, of 15 cases who were re-intubated, 11 (73\%) needed multiple re-intubations (seven, two, and two patients were re-intubated for once, twice, and tree times, respectively). All of these cases experienced hospital-acquired pneumonia, received atropine and 2-PAM for a time period up to 12 days and had a long hospital stay up to 30 days. All five patients who died belonged to this group, as well.

In our study, $80 \%$ of SEs occurred in the first 24 hours after tracheal intubation and $60 \%$ of them needed re-intubation in 24 hours. Epstein, et al. $[14,15]$ declared that all patients would need re-intubation within the first 72 hours. This may be due to the higher rate of SE in our patients which itself may be due to poor management of the doses of the sedatives or using atropine which results in agitation. Another reason may be the fact that 
all previous studies on this subject have been performed in general ICUs while our patients are OP-poisoned with severe respiratory effects [4].

2-PAM doses needed to be increased in those who underwent re-intubation probably because of respiratory failure secondary to intermediate syndrome. Respiratory muscle weakness due to IS was the cause of respiratory failure in four cases who received atropine and 2-PAM for 18 to 30 days. As shown, inappropriate dose of 2-PAM can lead to extubation failure and increase the need for re-intubation and prolonged MV in OP-poisoned patients. Mixed model analysis shows that there was not a statistically significant difference in atropine and 2-PAM doses between the two groups $(P=$ 0.441 and 0.381 , respectively).

There is a statistically significant correlation between decreased serum AchE level and increased need for re-intubation. Mixed model analysis showed that there was a statistically significant difference in the activity of AchE between those who were re-intubated and those who did not. Table 3 shows that although serum level of AchE was not related to the need for tracheal intubation at ED, there was a statistically significant correlation between decreases in AchE level and increasing the risk of re-intubation $(P=0.047)$.

\section{Sedative, physical restraint $\&$ agitation}

Although Bambi, et al. [7] believed that SE could be prevented with non-benzodiazepines drugs, use of BZDs is strongly recommended for OP poisoning [4,20]. Use of physical restrains without prescription of sedative drugs has not been recommended since it can be a risk factor for SE [7]. All of our patients had physical restraint but almost $50 \%$ self-extubated. This confirms that physical restraint in the presence of inappropriate and low doses of sedatives cannot prevent SE.

APACHE II score > 17, agitation, physical restraint, and higher levels of consciousness are major risk factors for SE [7]. Agitated patients are at greater risk of SE [15]. Therefore, the correct use of sedatives and education of the nursing staff can decrease these risks. Majority of OP-poisoned patients become alert a few hours after intubation and are at risk of SE in spite of ongoing respiratory compromise.

Early deep sedation and over-sedation are associated with worse outcomes and increased hospital mortality [21]. It seems that daily interruption of infusions of sedative drugs in comparison to continuous deep sedation can decrease the duration of MV and length of ICU stay $[21,22]$. On the other hand, inadequate sedation and uncontrolled agitation are the major risk factors for SE [9]. We used daily sedation interruption protocol for our patients by which nearly $50 \%$ of our patients self-extubated. Therefore, we believe that in cases with prediction of long need for MV (such as OP poisoning), sufficient sedative drugs should be prescribed specially in the first 24 hours of admission. Gradual decrease of the sedatives could prevent self-extubation [16]. In our study, of 15 patients who self-extubated, in six (40\%), weaning process had been started and the dose of sedatives had been waived. The patients had regained consciousness and removed their endotracheal tube. This means that nearly $40 \%$ of SEs happened when the physician started to reduce sedatives.

\section{Duration of MV, hospital stay, and outcome}

Although no relation exists between re-intubation and mortality, such a statistically significant relation exists between re-intubation and later complications $[20,23]$. Extubation failure will result in long-term hospitalization and increases mortality and later complications $[14,15]$.

SE may lead to increased duration of MV and hospital stay [7]. Although in this study, there was no strong correlation between duration of MV and SE, there was a significant correlation between SE and prolonged hospital stay (14.5 VS. 8.1 days, $P=0.048$ ). In our study, the mean time for MV and hospital stay was almost 2-fold in those who underwent re-intubation (9.3 days VS. 4.3 and 14.6 days VS. 8.2, respectively). This means that, early SE in inappropriate time can increase the duration of ICU stay in OP-poisoned patients and be a risk factor for poor outcome.

Of 31 patients who underwent tracheal intubation, 23 (75\%) needed MV more than 24 hours and 60\% underwent re-intubation. Mann Whitney test showed that a significant relation between the number of intubations and frequency of complications $(P=0.04)$. Hospitalization period was significantly longer in those who were re-intubated $(P=0.04)$.

Intermediate syndrome was the cause of respiratory failure and leading cause of re-intubation in 5 (16.1\%) cases; all of these patients had been re-intubated for one to three times and all of them needed prolonged MV and a long-time administration of atropine and 2-PAM and three of them died (3 of 5 dead cases). There was not statistically significant difference in rate of IS between those who were re-intubated and those who were not $(P=0.468)$. The mortality rate was not different between those with SE and PE but all five dead patients belonged to the group of the patients who underwent re-intubation $(P=0.011)$.

\section{Conclusion}

Outcome is poorer in the OP-poisoned patients who self-extubate. Rate of re-intubation is also higher in these cases. Re-intubation is related to longer hospitalization period, development of airway and pulmonary complications and increased mortality. Careful respiratory monitoring and administrating enough sedatives are recommended to prevent early unplanned SE in an inapposite time. 


\section{Acknowledgment}

We thank all fellows of medical toxicology and nurses who helped us with patient recruitment.

\section{Funding}

None.

\section{Conflict of Interest}

Authors declare that there is not financial relationships with any organization that might have an interest on this submitted article in the previous 3 years or any other relationships that could appear to have influenced this manuscript.

\section{References}

1. Li Z (2018) Evaluation of regulatory variation and theoretical health risk for pesticide maximum residue limits in food. J Environ Manage 219: 153-167.

2. Li Z (2018) Health risk characterization of maximum legal exposures for persistent organic pollutant (POP) pesticides in residential soil: An analysis. J Environ Manage 205: 163173.

3. Li Z (2018) Introducing relative potency quotient approach associated with probabilistic cumulative risk assessment to derive soil standards for pesticide mixtures. Environ Pollut 242: 198-208.

4. Xing XZ, Gao Y, Wang HJ, Qu SN, Huang CL, et al. (2015) Effect of sedation on short-term and long-term outcomes of critically ill patients with acute respiratory insufficiency. World J Emerg Med 6: 147-152.

5. Epstein SK (2002) Decision to extubate. Intensive Care Med 28: 535-546.

6. Menon N, Joffe AM, Deem S, Yanez ND, Grabinsky A, et al. (2012) Occurrence and complications of tracheal reintubation in critically ill adults. Respir Care 57: 1555-1563.

7. Bambi S, Rodriguez SB, Lumini E, Lucchini A, Rasero L (2015) Unplanned extubations in adult intensive care units: an update. Assist Inferm Ric 34: 21-29.

8. Fontenot AM, Malizia RA, Chopko MS, Flynn WJ, Lukan JK, et al. (2015) Revisiting endotracheal self-extubation in the surgical and trauma intensive care unit: Are they all fine? J Crit Care 30: 1222-1226.

9. Kiekkas P, Aretha D, Panteli E, Baltopoulos GI, Filos KS (2013) Unplanned extubation in critically ill adults: clinical review. Nurs Crit Care 18: 123-134.
10. Popat M, Mitchell V, Dravid R, Patel A, Swampillai C (2012) Difficult Airway Society Guidelines for the management of tracheal extubation. Anaesthesia 67: 318-340.

11. Bronstein AC, Spyker DA, Cantilena LR Jr, Green JL, Rumack BH, et al. (2008) 2007 Annual Report of the American Association of Poison Control Centers' National Poison Data System (NPDS): 25th Annual Report. Clin Toxicol (Phila) 46: 927-1057.

12. Kelly AM, Kerr D, Middleton P (2005) Validation of venous pCO2 to screen for arterial hypercarbia in patients with chronic obstructive airways disease. J Emerg Med 28: 377 379.

13. Corriols M, Marín J, Berroteran J, Lozano LM, Lundberg I, et al. (2008) The Nicaraguan Pesticide Poisoning Register: constant underreporting. Int J Health Serv 38: 773-787.

14. Epstein SK, Nevins ML, Chung J (2000) Effect of unplanned extubation on outcome of mechanical ventilation. Am J Respir Crit Care Med 161: 1912-1916.

15. Epstein SK (2002) Extubation. Respir Care 47: 483-492.

16. Kulkarni AP, Agarwal V (2008) Extubation failure in intensive care unit: predictors and management. Indian $\mathrm{J}$ Crit Care Med 12: 1-9.

17. Da Silva PS, Fonseca MC (2012) Unplanned endotracheal extubations in the intensive care unit: systematic review, critical appraisal, and evidence-based recommendations. Anesth Analg 114: 1003-1014.

18. Jarachovic M, Mason M, Kerber K, McNett M (2011) The role of standardized protocols in unplanned extubations in a medical intensive care unit. Am J Crit Care 20: 304-311.

19. Jaber S, Chanques G, Altairac C, Sebbane M, Vergne C, et al. (2005) A prospective study of agitation in a medical-surgical ICU: incidence, risk factors, and outcomes. Chest 128: $2749-2757$.

20. Thille AW, Harrois A, Schortgen F, Brun-Buisson C, Brochard $L$ (2011) Outcomes of extubation failure in medical intensive care unit patients. Crit Care Med 39: 2612-2618.

21. Tanaka LM, Azevedo LC, Park M, Schettino G, Nassar AP, et al. (2014) ERICC study investigators. Early sedation and clinical outcomes of mechanically ventilated patients: a prospective multicenter cohort study. Crit Care 18: 156.

22. Treggiari MM, Romand JA, Yanez ND, Deem SA, Goldberg $\mathrm{J}$, et al. (2009) Randomized trial of light versus deep sedation on mental health after critical illness. Crit Care Med 37: 2527-2534.

23. Vale A, Lotti M (2015) Organophosphorus and carbamate insecticide poisoning. Hangb Clin Neurol 131: 149-168. 